\title{
Supporting Information Out-of-Equilibrium Measurements of Kinetic Constants on a Biosensor
}

\author{
Donatien Mottin, ${ }^{\dagger} \ddagger$ Florence Razan, ${ }^{\ddagger}$ Claude Nogues, ${ }^{\top}$ and Marie-Caroline \\ Jullien ${ }^{*} \dagger$ \\ $\dagger$ Univ. Rennes 1, CNRS, IPR (Institut de Physique de Rennes) UMR 6251, F-35000 \\ Rennes \\ $\ddagger$ ENS Rennes, SATIE, UMR-CNRS 8029, Campus de Ker Lann, F-35170 Bruz \\ IENS Paris-Saclay, LBPA UMR-CNRS 8113, Gif-sur-Yvette, France \\ E-mail: marie-caroline.jullien@univ-rennes1.fr \\ Phone: +33223237852
}

\section{Contents}

DNA Sequences

S1

Numerical Procedures

S1

Convection-Diffusion Model of a Sample Plug

S3

\section{DNA Sequences}

The DNA sequences (Eurogentec) were randomly generated, with verification of the absence of self-complementarity. The fixed DNA strand is 50 base long and have a $\mathrm{SH}$ function at its 5 ' end, while the free DNA strand is 26 base long, which are complementary to the 26 bases at the 3' end of the fixed DNA.

Free DNA strand: 5'-CGA GTC GCA AGA TGG CGG ATC CGA GTG ACA GAT GG-3'

Fixed DNA strand: SH-C6H12- T5-5' CCA TCT GTC ACT CGG ATC CGC CAT CTT GCG ACT CG-3'

\section{Numerical Procedures}

We first present the geometry that mimics the experimental system which is considered in the simulations, see Figure S1. This geometry is composed of three sequential coaxial cylinders of radius $R$. The cylinder $\mathrm{A}$ represents the initial sample plug, of length $L_{0}=\frac{\pi R^{2}}{V_{0}}$. It contains an inlet where fluid is injected at a flow rate $Q$ and one outlet connected to the cylinder $\mathrm{B}$ while its side wall is a rigid non-reactive surface. The cylinder $\mathrm{B}$ is of length $L_{1}$ and represents the fluidic connections between the initial plug formation zone and the microfluidic system. It contains an inlet connected to cylinder $\mathrm{A}$, and an outlet connected to cylinder $\mathrm{C}$. Its side wall is also a rigid non-reactive surface. The cylinder $\mathrm{C}$, of length $L_{1} / 100$, mimics the microfluidic system. It contains an inlet connected to cylinder B and an outlet. Its side wall is a sensitive surface with an initial ligand concentration $s_{m}$, a binding constant $k_{\text {on }}$ and a dissociation constant $k_{\text {off }}=0$. The binding kinetics follows the first-order Langmuir kinetics described in this paper.

The geometrical model of the cylinder $\mathrm{C}$ does not exactly reproduce the experimental geometry. This can be justified by considering that the complex formation is limited by the complexation kinetics on the biochip surface, and not by the mass transport (diffusion and flow) towards it. Under this assumption, as explained 


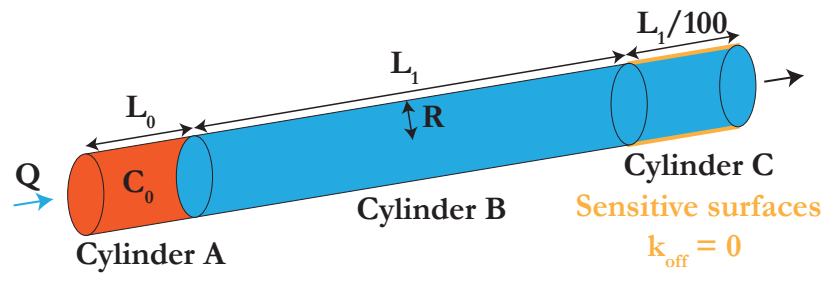

Figure S1: Numerical initial geometry for both COMSOL and Lagrangian simulations. The sample is initially located in the cylinder $\mathrm{A}$, at a concentration $c_{0}$, while cylinders $\mathrm{B}$ and $\mathrm{C}$ are free from targets. Since $t=0$, a flow rate $\mathrm{Q}$ (with no extra targets) enters the system by the cylinder A and pushes the sample. The side wall of the cylinder $\mathrm{C}$ is sensitive and mimics the biochip surface. Target complexation follows equation (1) of the main text.

by Squires et al., ${ }^{1}$ the geometry has no influence on the capture rate. This numerical geometry simplifies the problem by greatly facilitating numerical convergence and reducing the resolution time.

In a first approach, COMSOL Multiphysics $\mathbb{R}$ simulations are carried out. In this simulation, the problem is described in term of a target concentration field (generally called Eulerian description). The boundary conditions that are considered are: injection of a target-free liquid at a flow rate $Q$ at the cylinder A inlet and zero pressure at the cylinder $\mathrm{C}$ outlet. Initial conditions are a zero concentration field everywhere, except in the cylinder A where the concentration equals $c_{0}$. This plug sample flows through cylcinder B and reaches cylinder C. During this travel, the sample plug is distorted following Taylor-Aris dispersion and reaches cylinder C with a diluted concentration $c$. At the biochip surface, the complexation rate is computed using equation (1) of the main text, taking the local concentration field value $c$. The numerical procedure is the following: steady-state laminar flow solution is first computed, and the temporal transport solution is then computed taking into account diffusion, convection and surface complexation. The mesh is gradually refined until the convergence of the solution is guaranteed. The grey points of Figure 5 of the main text represents the results of these simulations.
In a second approach, we performed other simulations, using a code made in our laboratory. Instead of describing the concentration field (mean field approach)), the trajectory of each target is computed (generally called Lagrangian description). The cylinder $\mathrm{A}$ is initially filled with point objects at a concentration $c_{0}$ and of diffusion coefficient $D$. A Poiseuille velocity profile $V(\vec{r})$ is computed analytically using $Q$ and $R$. At each time step $d t$, the equation of motion is solved for each object. First, the objects are convected by the surrounding liquid over a distance $V(\vec{r}) d t$. Then, the objects diffuse. Diffusion is simulated by choosing a random direction in 3D along which the object moves over a length $\sqrt{6 D d t}$. If the objects get out of the simulation volume, we use the mirror boundary condition to bring them back inside the volume. The only exception is if an object leaves the simulation volume through the outlet of the Cylinder $\mathrm{C}$, in which case it is removed from the simulation. Finally, if the object is inside the cylinder $\mathrm{C}$ and its distance from the side wall is less than the diffusion length $\sqrt{6 D d t}$ (which means that it probably hits the wall by a random walk during the time $d t$ ), a random binding is tested: the object binds with a probability $\xi=k_{\mathrm{on}} s_{m} \frac{d t}{6 D}$ (see details at the end of this section). The accuracy of the simulation is ensured by sufficient refinement of the time step $d t$ : 1) a target needs several time steps to travel through all the channels (convection is not under-resolved): $\left.\max (V) \ll \frac{L_{1}+L_{0}}{d t} ; 2\right)$ a target diffuses over the cross-section of the channels in several time steps (transverse diffusion is not under-resolved): $\sqrt{6 D d t} \ll R ; 3$ ) a target diffuses along the channels in several time steps (axial diffusion is not under-resolved) $\sqrt{6 D d t}<L_{1} / 100$ : and 4) all targets near the sensor do not adhere at the same time (reaction is not under-resolved): $\xi \ll 1$. The blue points of Figure 5 of the main text represents the results of these simulations.

It should be noted that the Lagrangian simulations were carried out targets by targets. An important consequence is that the realization is only possible at low concentration to limit the computation time. Thus the $k_{\text {on }}$ that have been 
implemented vary from $10^{-12}$ to $10^{-8} \mathrm{~m}^{3} \mathrm{~mol}^{-1}$ $\mathrm{s}^{-1}$. It is clear that it is not the absolute value of $k_{\text {on }}$ that counts for these simulations, but its relative value with the value obtained at the output of the calculation. In the end, this does not change the universality of the law obtained.

\section{Computation of the Random Binding Probability $\xi$}

In the Lagrangian simulation, we need to convert the statistical binding equation of the main text:

$$
\frac{d s}{d t}=k_{\mathrm{on}} c_{0} s_{m}
$$

into a discrete binding probability $\xi$, tested for each targets in the vicinity of the biochip. According to equation (1), during a time $d t$ the number of targets which binds to a biochip of surface $S$ is:

$$
d N=k_{\mathrm{on}} c_{0} s_{m} S d t
$$

On the other hand, in the Lagrangian simulations, the binding probability is tested on the $N$ targets that can reach the biochip by doing a diffusion step $\sqrt{6 D d t}$ during $d t$ :

$$
N=c_{0} S \sqrt{6 D d t}
$$

Thus, to mimic equation (1) with the correct value of $k_{\text {on }}$, the $\xi$ value should be chosen so that $d N$ targets out of $N$ bind to the surface during a time $d t$. Thus:

$$
\xi=\frac{d N}{N}=k_{\mathrm{on}} s_{m} \sqrt{\frac{d t}{6 D}}
$$

\section{Convection-Diffusion Model of a Sample Plug}

In this section, we derive the equation (7) of the main text:

$c=c_{0} \operatorname{erf}\left(\frac{L_{0}}{4 \delta}\right)$ with $\delta=\sqrt{\frac{D_{\mathrm{TA}}\left(L_{1}+L_{0} / 2\right) \pi R^{2}}{Q}}$

The concentration above the biochip is taken while the sample barycenter reaches it (yellow part of the corresponding sensorgram of Figure $2 c$ in the main text).

\section{Geometry and Axis Definitions}

The geometry of the computation is sketched on Supplementary Figure S2.

We consider a tube of length $L_{0}+L_{1}$ and of radius $R . \quad x$ is the axis parallel to the tube, in the laboratory frame of reference, oriented from the entry to the end. The origin is located at the initial position of the sample barycenter. $X$ is associated to the sample barycenter at all time.

Under to Taylor-Aris assumption, $t \geq \frac{D}{R^{2}}$, the concentration depends only on $x$ and $t$, as diffusion homogenizes the concentration in the cross-section. The problem thus reduces to a $1 \mathrm{D}$ diffusion problem, with an effective diffusion coefficient $D_{T A}$.

In the following, we first compute the diffusion in the $X$ frame of reference and then and then come back to the $x$ laboratory frame of referecne to take convection into account.

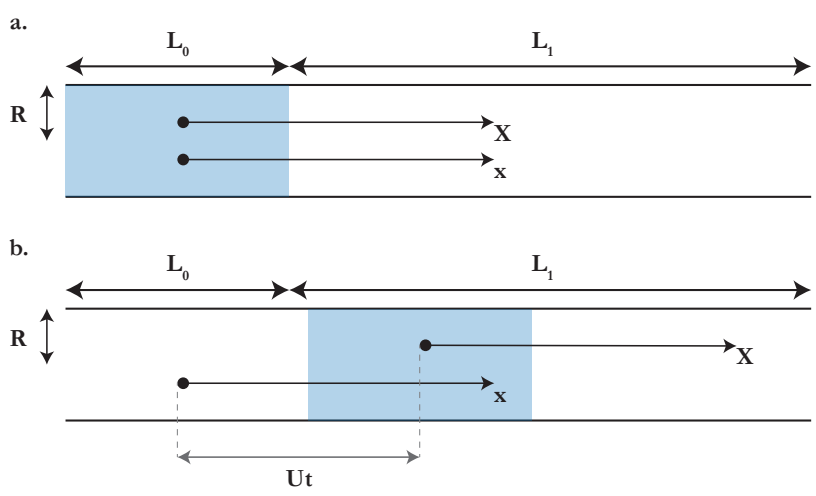

Figure S2: Computation Geometry and Axis Definition. The blue area is a schematic view of the sample. Its dispersion is not represented. a. At $t=0$, the $x$ and $X$ axis are identical. b. At $t>0$, the two axis are shifted by $U t$.

\section{Diffusion in the sample frame of reference}

At $t=0$, the concentration profile is: 


$$
c(X, t=0)= \begin{cases}c_{0} & \text { if } X \in\left[-L_{0} / 2 ; L_{0} / 2\right] \\ 0 & \text { otherwise }\end{cases}
$$

A diffusive point in $1 \mathrm{D}$, located in $X_{0}$, spreads following the law:

$$
c_{X_{0}}(X, t)=\frac{A}{\sqrt{D_{\mathrm{TA}} t}} \exp \left(-\frac{\left(X-X_{0}\right)^{2}}{4 D_{\mathrm{TA}} t}\right)
$$

Where $A$ is a normalization factor. Thus, to obtain the complete profile, one has to integrate over all the sources, between $-L_{0} / 2$ and $L_{0} / 2$ :

$$
\begin{aligned}
c(X, t) & =\int_{-L_{0} / 2}^{L_{0} / 2} c_{X_{0}}(X, t) d X_{0} \\
& =\frac{A}{\sqrt{D_{\mathrm{TA}} t}} \int_{-L_{0} / 2}^{L_{0} / 2} \exp \left(-\frac{\left(X-X_{0}\right)^{2}}{4 D_{\mathrm{TA}} t}\right) d X_{0}
\end{aligned}
$$

Which gives, after normalization and using erf imparity:

$c(X, t)=\frac{c_{0}}{2}\left[\operatorname{erf}\left(\frac{L_{0} / 2-X}{2 \sqrt{D_{\mathrm{TA}} t}}\right)+\operatorname{erf}\left(\frac{L_{0} / 2+X}{2 \sqrt{D_{\mathrm{TA}} t}}\right)\right]$

\section{Convection: Laboratory Frame of Reference}

In the laboratory frame of reference $x=X+U t$ with $U=\frac{Q}{\pi R^{2}}$, leading to:

$c(x, t)=\frac{c_{0}}{2}\left[\operatorname{erf}\left(\frac{L_{0} / 2-x+U t}{2 \sqrt{D_{\mathrm{TA}} t}}\right)+\operatorname{erf}\left(\frac{L_{0} / 2+x-U t}{2 \sqrt{D_{\mathrm{TA}} t}}\right)\right]$

With our geometrical definitions, the barycenter of the sample reaches the biochip after traveling a distance $x=L_{0} / 2+L_{1}$ during a corresponding time $t=\frac{L_{0} / 2+L_{1}}{U}$ :

$$
c^{*}=c\left(\begin{array}{l}
x=L_{0} / 2+L_{1} \\
t=\frac{L_{0} / 2+L_{1}}{U}
\end{array}\right)
$$

$$
c^{*}=c_{0} \operatorname{erf}\left(\frac{L_{0}}{4} \sqrt{\frac{U}{D_{\mathrm{TA}}\left(L_{0} / 2+L_{1}\right)}}\right)
$$

By injecting the expression of $U$, we see that:

$\sqrt{\frac{U}{D_{\mathrm{TA}}\left(L_{0} / 2+L_{1}\right)}}=\sqrt{\frac{Q}{D_{\mathrm{TA}} \pi R^{2}\left(L_{0} / 2+L_{1}\right)}}=\frac{1}{\delta}$

Which leads to:

$$
c=c_{0} \operatorname{erf}\left(\frac{L_{0}}{4 \delta}\right)
$$

\section{References}

(1) Squires, T. M.; Messinger, R. J.; Manalis, S. R. Nat. Biotechnol 2008, 26, 417. 